\title{
Gear dynamics monitoring using discrete wavelet transformation and multi-layer perceptron neural networks
}

\author{
Javier Sanz Ricardo Perera Consuelo Huerta
}

\begin{abstract}
A B S T R A C T
This paper presents a multi-stage algorithm for the dynamic condition monitoring of a gear. The algorithm provides information referred to the gear status (fault or normal condition) and estimates the mesh stiffness per shaft revolution in case that any abnormality is detected. In the first stage, the analysis of coefficients generated through discrete wavelet transformation (DWT) is proposed as a fault detection and localization tool. The second stage consists in establishing the mesh stiffness reduction associated with local failures by applying a supervised learning mode and coupled with analytical models. To do this, a multi-layer perceptron neural network has been configured using as input features statistical parameters sensitive to torsional stiffness decrease and derived from wavelet transforms of the response signal. The proposed method is applied to the gear condition monitoring and results show that it can update the mesh dynamic properties of the gear on line.
\end{abstract}

\section{Introduction}

The ability to achieve accurate prognostics is critical to the optimal maintenance of rotating machinery with the purpose of avoiding the high costs associated with machine downtime in the manufacturing industry. A suitable advance warning system would allow for the scheduling of timely corrective and repair actions as well as extending the maintenance cycles. The trade-off associated with implementing such a system is that it requires a sensing system to be deployed on the machine and a sophisticated data analysis procedure that can be used to interrogate the measured data. Through this system the existence and location of damage should be detected.

On the other hand, the estimation of the engineered system's remaining useful life and the machine performance when damage appears, would require not only the process of identifying the presence of damage but also either quantify the extent of damage or update the current gear dynamics based on information extracted from the measured system response. This would require a more sophisticated method based on statistical procedures.

Considerable research has been carried out previously for the development of various techniques for gear fault detection and diagnosis based on vibration. These techniques can be classified into time domain, frequency domain and time-frequency domain.
Analyzing the vibration signals directly in the time domain is one of the simplest detection and diagnosis approaches. Various time domain statistical parameters can be used as trend parameters to detect the presence of damage [1]. The time synchronous average (TSA) providing an average time signal of one individual gear over a large number of cycles has also been acknowledged as a powerful and very successful tool in the detection of gear faults $[2,3]$ since it can remove the background noise and all the periodic events that are not exactly synchronous with the gear of interest.

In the frequency domain approach the dominant frequency components of the vibration signals and their amplitudes, together with sidebands due to modulation phenomena, are used for trending purposes. As faults occur, the corresponding amplitudes of the peaks in the power spectrum increase as well as the number and amplitude of the sidebands. However, the spectral analysis may be unable to detect gear failures in the case of local faults, which primarily affect sidebands, due to the difficulty of evaluating the spacing and evolution of sideband families in the spectrum.

Another solution of the problem is offered by a time-frequency distribution, which is a function giving a distribution of the total energy of the signal at particular time and frequency points. The most common approach for performing this kind of analysis is the short-time Fourier transform (STFT) based on applying the Fourier transform (FT) to sections or windows of a signal and by translating the window along the signal of interest. The width of the window is kept constant throughout the analysis and specifies the resolutions of time and frequency information. Common forms of this window include the Hanning or Gaussian functions. The first 
work in this area goes back to Gabor [4] and Hoelström [5]. Other different methods for extracting a time-frequency map of a signal are the Wigner-Ville distribution (WVD) [6] and the Choi-Williams distribution [7].

Decompositions based on FT do not give any local information about the function due to the infinite support of the trigonometric functions used in the analysis. Wavelet transforms (WT), however, provide for locality both in time and frequency. Originally developed at the end of the 80s [8,9], wavelet transforms have been gaining popularity for their capability to treat transient signals and have been increasing interest in recent years as a tool for fault detection both in machinery [10-12] and in civil engineering structures [13,14] and for other engineering fields [15].

Through the preprocessing of the vibration signals using discrete wavelet transform (DWT), a monitoring of the signals in the time-frequency domain is obtained which can offer either warnings or localizations of the perturbations.

The method above makes it possible to identify and locate faults at early stages in the monitored machine. However, little study has been developed about the on-line estimation of the changes involved in dynamic mesh stiffness when a fault is detected and localized. This updating task is necessary to assess the structural integrity of the gear and the rest of the machine.

This mesh stiffness is a key parameter in the gear dynamics because it has a direct impact on load-carrying capacity of the gear, vibrations induced and dynamic tooth loads. Many studies have been performed in the past to determine the influence of the aforementioned mesh compliance in gear dynamics $[16,17]$. This study aims to detect this torsional stiffness change on-line which implies an extra feature over such techniques $[16,17]$. In addition the evaluation on line of the change in stiffness allows to determine its influence over the gear operating point which can be critical in some applications due to the fact that changes in mesh stiffness associated with some failures can decrease the frequency value of some resonances, with the potential risk of interacting with another exciting frequencies in the rotary machine, like shaft unbalances, giving raise to unforeseen changes that should be evaluated either for maintenance purposes and to avoid unexpected failures.

Moreover with this updated dynamic information the new potential risk operation points (new critical torque and speed conditions) in a rotary machine can be avoided when a perturbation is detected in the response signal.

Therefore, in this work, one step forward is given with the purpose of determining the mesh stiffness changes when a failure appears per shaft revolution. To this end, a two-stage algorithm, which combines DWT (discrete wavelet transformation) preprocessing and MLPN (multi layer perceptron neural network) prognostics, has been developed. In previous publications, rotating machinery fault diagnosis tools based on soft computing techniques have also been developed [18-21]. In most cases, a first stage of feature selection or preprocessing is implemented with the purpose of serving as input, in a second stage, to a feature-based classifier for fault diagnosis. In the proposed methodology, the first stage, based on DWT, serves by itself as a fault diagnosis tool and as preprocessor for a second stage in which fault severity is estimated. According to this, the first stage plays also as a filter since only those signals detected as faulted in the first stage are fed to the second stage.

Mathematical models of gear systems can be used in order to study the effects of faults on gear torsional vibrations with the purpose of developing more suitable techniques for monitoring, diagnostics and quantification. A review of spur gear dynamic models with and without defects has been done by Ozguben and Houser [16] and Parey and Tandon [22], respectively. In this work, a local damage in one tooth has been simulated by assuming a modulation function in mesh stiffness [23]. It should be remarked that
1.Tangential acceleration time recording (shaft revolution)

Discret wavelet coefficients generation. STAGE 1

3. Wavelet coefficients abnormality checking. STAGE 1

4. Wavelet coefficients statistical parameter generation.

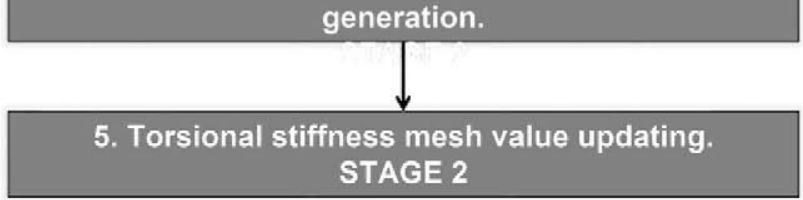

Fig. 1. Scheme of the proposed method.

with the new proposed algorithm, the diagnosis referring either to warning or localization or stiffness reduction is provided per shaft revolution. Furthermore, the proposed procedure allows performing a dynamic evaluation on-line that can predict changes in rotating machine resonances.

The layout of the paper is as follows. In Section 2, the scheme for machine health assessment is presented as generalized technique to detect and on-line update the gear dynamics. In Section 3 , a short description of the machine model used for the study is performed. In the following section, the implementation of the first stage of the algorithm is analyzed emphasizing the advantages of using DWT as a pre-processing tool when compared with traditional techniques. In Section 5, implementation of the second stage of the proposed is presented in order to estimate the mesh stiffness reduction once the fault has been identified. At the end of the paper we will summarize the present study with the final conclusions.

\section{Method for rotating machinery health assessment}

The schematic representation of the proposed system is shown in Fig. 1. The scheme consists mainly of two major parts or stages, namely (i) data acquisition and signal processing by DWT for identifying machine faults and (ii) post-processing and fault severity diagnosis by using a pattern recognition tool like NNs. The scheme can be implemented as an on-line assessment of machine health.

The first stage consists in three steps (steps 1-3), namely (1) data acquisition with accelerometers, (2) time signal processing with discrete wavelet transform and (3) inspection of the transformed signal to detect abnormalities. By using DWT the time signal is zoomed which allows showing the changes in torsional mesh stiffness when the gear is faulted. This first stage plays as a filter, since if there is not detection of abnormalities in the first stage the procedure jumps again to the step 1 . However, if any abnormality is detected, the algorithm continues with the following steps in a second stage (steps 4 and 5 ) of fault diagnosis. By using sensitive statistics for a particular decomposition level of the post-processed DWT signal (step 4), the time data are used as input to an artificial neural network (step 5), previously trained, which finally tracks online the value of the gear mesh stiffness and, therefore, the severity of fault. Although combination of DWT coefficients as input values to a MLPN has been used in other gear applications [24,25] their scope normally is limited to damage detection and it is unusual to find a direct application to gear dynamic updating on line. 


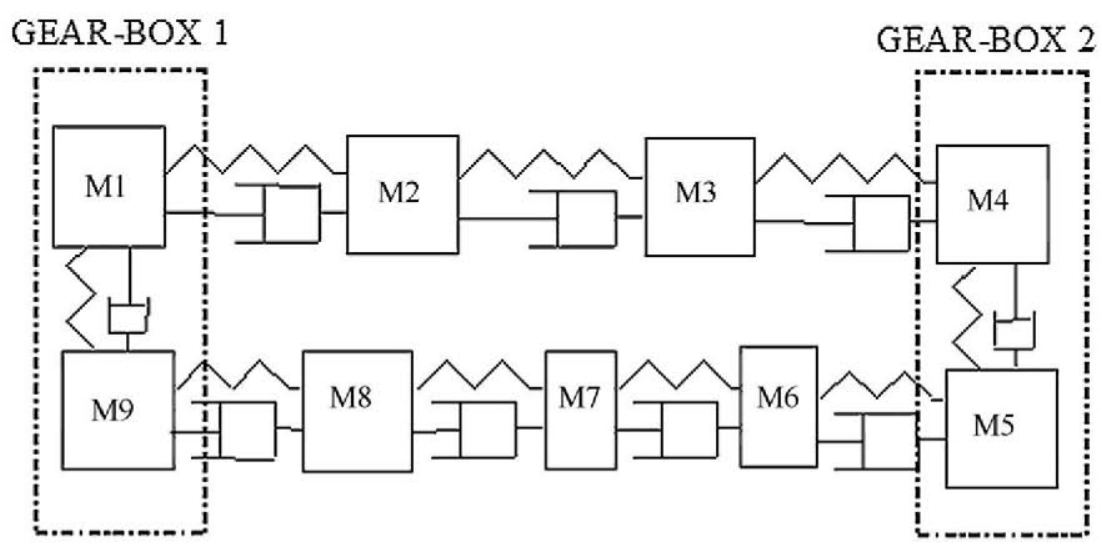

Fig. 2. Lumped parameter model.

The on-line implementation of the assessment procedure of bearing health is possible once the off-line modeling has been performed, i.e., when a proper DWT decomposition level has been fixed and when the neural network has been trained. This requires the use of historic data from normal and faulty bearings. In this sense, although the off-line modeling is clearly dependent on each particular gear dynamics, the general scheme of the proposed algorithm (Fig. 1) can be analogously implemented in any gear machine. To do it, logically, a previous off-line modeling of each machine should be performed.

\section{Description of the elastodynamic model}

To evaluate the proposed procedure in Section 3, a specific gear machine, briefly described below, has been taken as reference.

Gear boxes are one of the most common components in modern rotating machinery; being able to detect accurately the existence and severity of a fault in a machine can be of prime importance for taking decisions automatically, and reliably on the running health of a machine. All the studies in this work use vibration data taken from the mathematical model of the gear system reported by Dalpiaz et al. [26]. The gear machine is composed of two identical single-stage gear boxes mounted back to back, with a locked-in torque. Each gear unit is constituted by a carburized spur gear pair of module $3 \mathrm{~mm}$; the pinion and the wheel have 28 and 55 teeth, respectively. Further details about the gears can be found in [27].

\subsection{Mathematical model}

In order to simulate the vibrational characteristics of this machine, a non-linear torsional model was used (Fig. 2). In the proposed model, only the torsional stiffness, represented by linear springs, of the bearings and the shafts were considered, resulting in nine torsional DOFs. Analogously, translating masses were represented by the rotational inertias. Furthermore, dampers of constant value and proportional to the corresponding spring stiffness were put between every two masses.

All the vibrational signals generated numerically in this paper correspond to a wheel torque of $1139 \mathrm{Nm}$ and to wheel and pinion speeds of $254.5 \mathrm{rpm}$ and $500 \mathrm{rpm}$, respectively; thus the meshing frequency is $233.3 \mathrm{~Hz}$. The generated signals were assumed to represent the measured tangential accelerations in the base circle of the wheel of gearbox corresponding to the mass connected to the degree of freedom labeled with ' 9 ' in Fig. 2. Furthermore, a white noise of mean ' 0 ' and power ' $0.1(\mathrm{Nm})^{2} / \mathrm{Hz}$ ' was added to the input torque. For this background noise the signal to noise ratio (SNR), defined as:

$\mathrm{SNR}=\left(\frac{A_{\text {signal }}}{A_{\text {noise }}}\right)^{2}$

takes a value of 126.8 , where $A_{\text {signal }}$ and $A_{\text {noise }}$ are the root mean square (RMS) amplitude for the signal and the noise, respectively.

The noise influence over the response signals (corresponding to damage and non damaged scenarios) will be evaluated in Section 4.

Mesh stiffness variability has been considered to be the main source of gear vibration. The mesh stiffness model assumes that the design, manufacturing and assembly errors are not taken into account and, furthermore, the transmission error is due only to the variation in the position of the meshing point along the line of action.

Consequently a vibration signal generated by the mesh action mentioned above should be expected. It is common to represent this signal as [23]:

$y(t)=\sum_{r=1}^{R} X_{r} \cos \left(2 \pi Z f_{S} r t+\phi(t)\right)$

where $R$ is the number of harmonics considered, $Z$ is the number of teeth of the gear and $X_{r}$ and $\Phi(t)$ represent the amplitude and phase modulation of the $r$ th harmonic, respectively. On the other hand, $t$ is the time analyzed and $f_{s}$ is the shaft frequency.

It should be emphasized that, according to Eq. (2), although we had a perfect gear without manufacturing or assembly errors there will always be a source of vibration induced by the teeth engaging action.

Variable mesh stiffness should be incorporated in the model in order to guarantee a vibration signal as depicted in Eq. (2). In case of considering only the fundamental harmonic in Eq. (2), the meshing stiffness would be simulated by using a single sinusoid (Fig. 3).

The lumped mathematical model was built using the Simulink interface from MATLAB and the differential equations of motion of this model were solved using the Dormand-Prince finite difference method [28].

Fig. 4 shows a time history of the tangential acceleration numerically obtained under the operating conditions specified above. In spite of its simplicity, the torsional model has similar acceleration values that the ones obtained experimentally and numerically by Dalpiaz et al. [26].

It should be remarked that the main purpose of this work is using the torsional model as a "virtual machine" to introduce faults of 


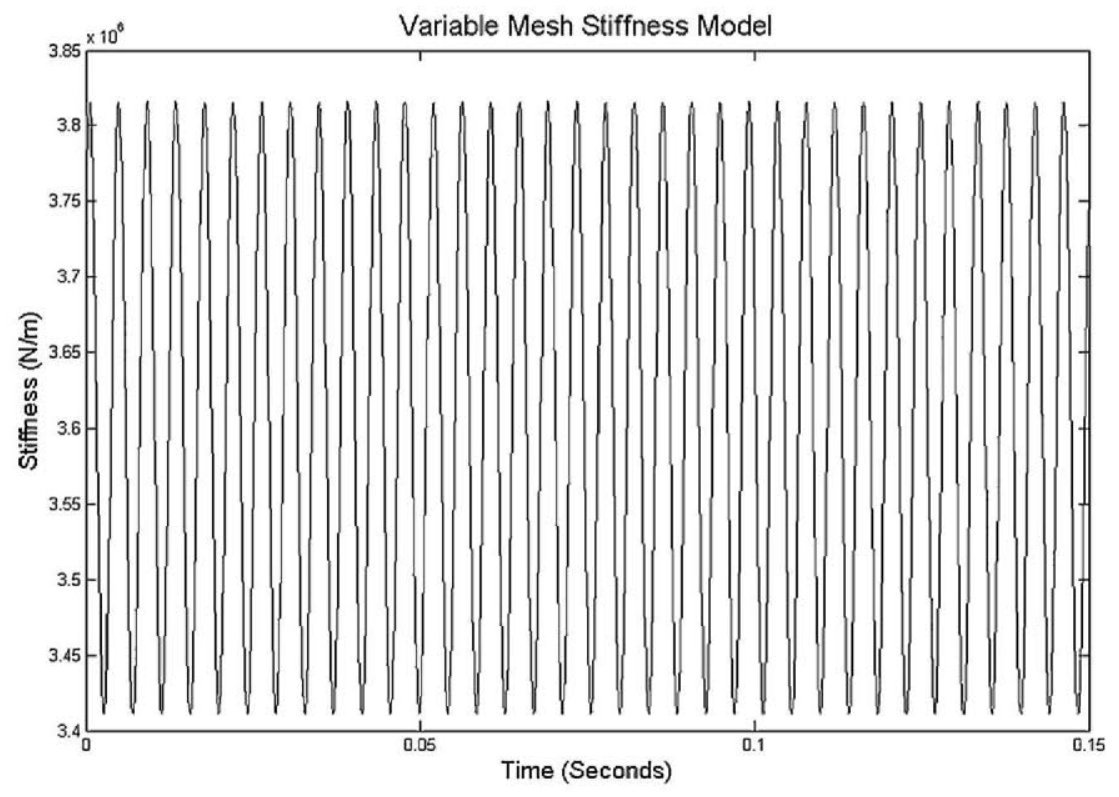

Fig. 3. Variable mesh stiffness model.

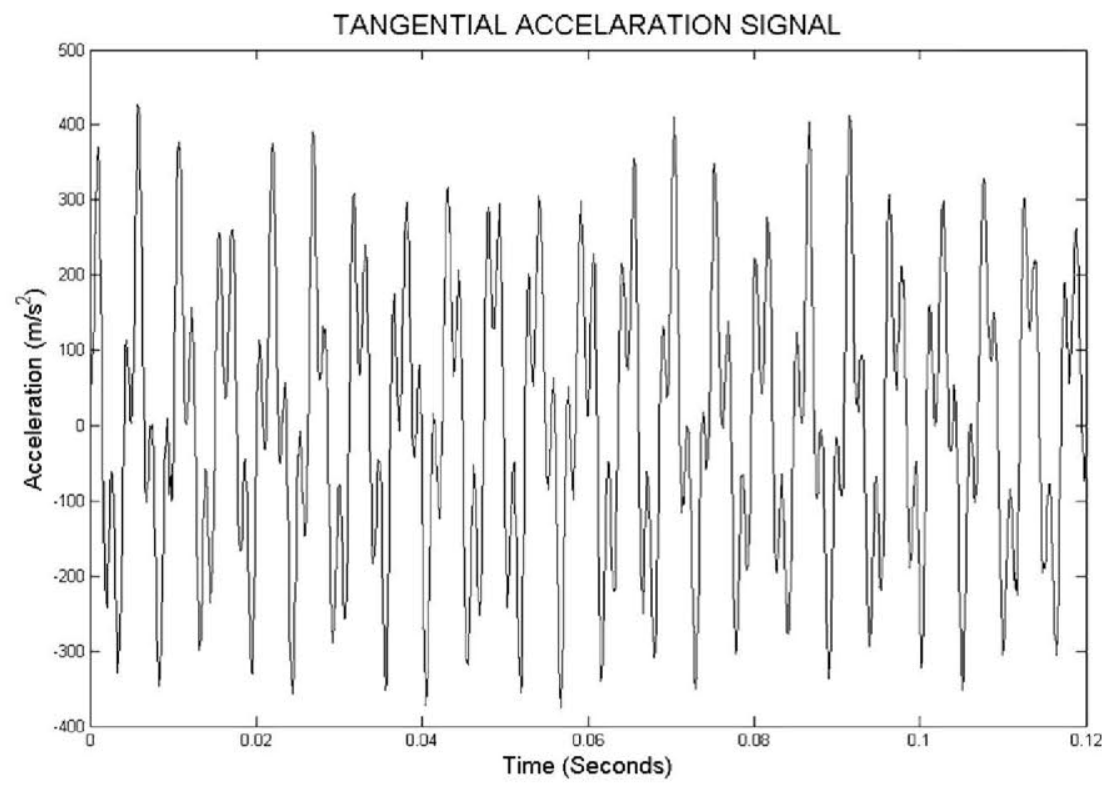

Fig. 4. Numerical tangential acceleration in the time domain.

different severity that allow checking the procedure of identification and dynamic updating presented later.

\subsection{Damage model}

Local faults in gears are considered in this study and the way of introducing their effect in a global mathematical expression will be depicted next.

Local damage in gear teeth generates a local decrease in mesh stiffness [17]; as a consequence the damage in gears produce modulation effects modifying either the magnitude or the phase of the vibration signal picked up by the transducers. These effects occur during the engagement of the faulted teeth, but are repeated once per revolution of the gear. In order to study the modifications in torsional vibrations due to faults, one damaged tooth is considered whose cause might be due to phenomena such as spalls in tooth flank and cracks in tooth root. The algorithm developed in this study might be applied to any local gear failure because most of them have in common the torsional mesh stiffness reduction when the affected area is contacting with the mating tooth. The modulation phenomena due to gear faults are modeled through amplitude and phase modulation functions, $a_{r}(t)$ and $p_{r}(t)$, respectively, given by the expressions:

$$
\begin{aligned}
& a_{r}(t)=\sum_{m=1}^{M} a_{m r} \cos \left(2 \pi r f_{s} t+\alpha_{m r}(t)\right) \\
& p_{r}(t)=\sum_{m=1}^{M} p_{m r} \cos \left(2 \pi r f_{s} t+\beta_{m r}(t)\right)
\end{aligned}
$$

where $M$ is the number of sidebands around the $r$ th tooth meshing harmonic, and $a_{m r}(t)$ and $p_{m r}(t)$ are the magnitude of the amplitude and phase functions, respectively, associated with the $r$ th order and 


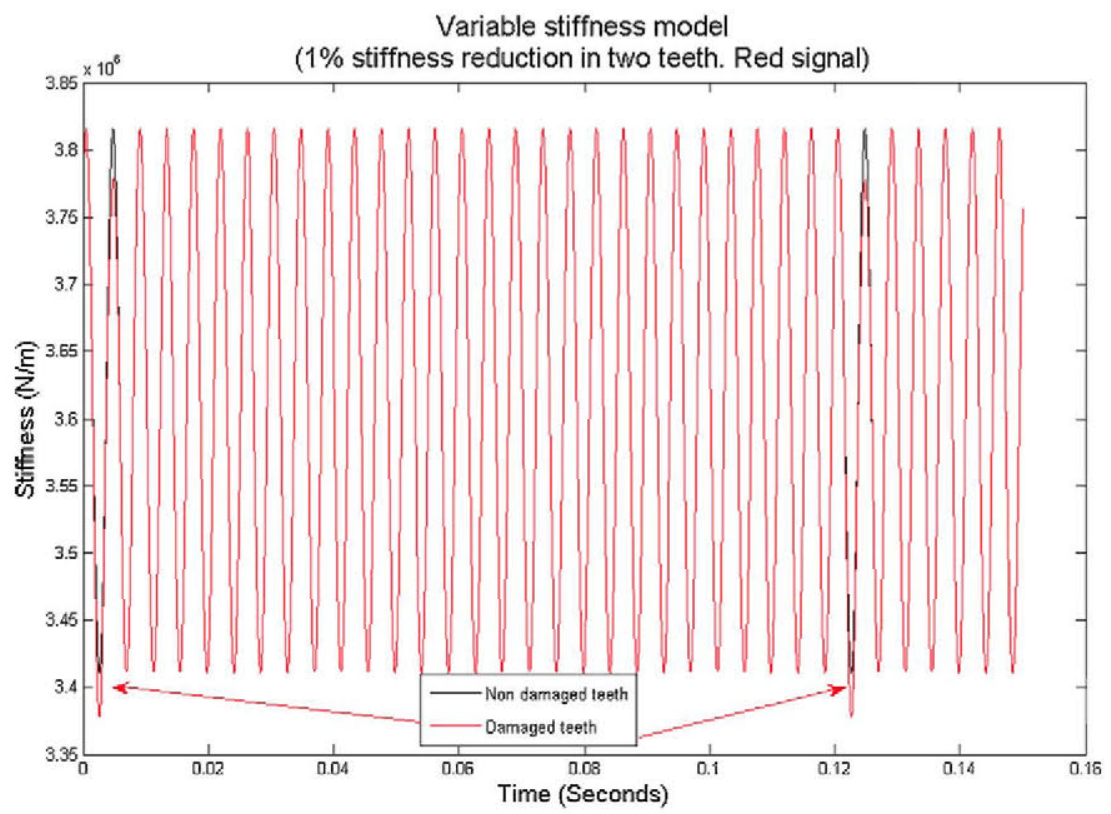

Fig. 5. Variable mesh stiffness model used in case of damage.

the $m$ th sideband. Finally, $\alpha_{m r}(t)$ and $\beta_{m r}(t)$ represent the phases of both modulating functions for the $m$ th sideband of $r$ th order.

The final expression that gathers the source of vibration generated only by mesh action (Eq. (2)) and the modulation functions (Eqs. (3) and (4)) produced by gear faults can be combined through the following expression [23]:

$y^{\prime}(t)=\sum_{r=1}^{R} X_{r}\left(1+a_{r}(t)\right) \cos \left(2 \pi Z f_{s} r t+\phi(t)+p_{r}(t)\right)$

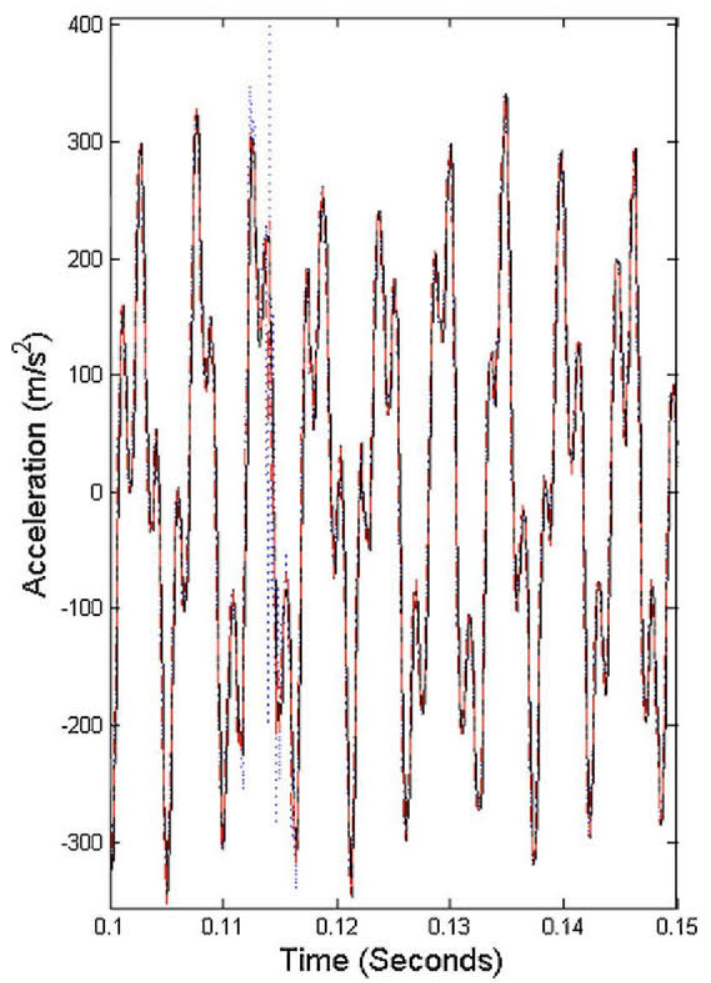

where all the parameters have been defined previously.

In order to introduce the gear fault in our model, the mesh stiffness is modified according to Eq. (5), considering only the first harmonic (the mesh frequency). Moreover in order to avoid discontinuities in the mesh stiffness, only the magnitude modulation effect is considered since the discontinuities introduced by using a phase modulation generate huge fluctuations of the acceleration response signal, which are far from the real conditions.

Consequently, the modified mesh stiffness corresponding to a damaged gear appears to be as shown in Fig. 5 .

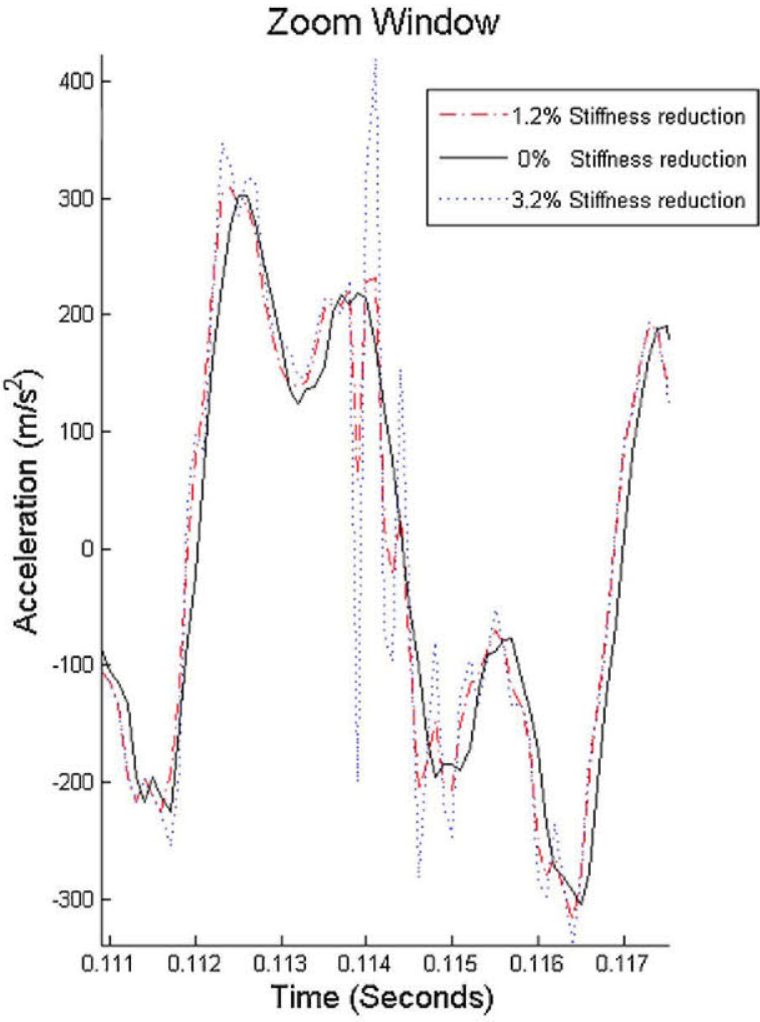

Fig. 6. Numerical tangential acceleration in the time domain for the damaged and healthy cases. 


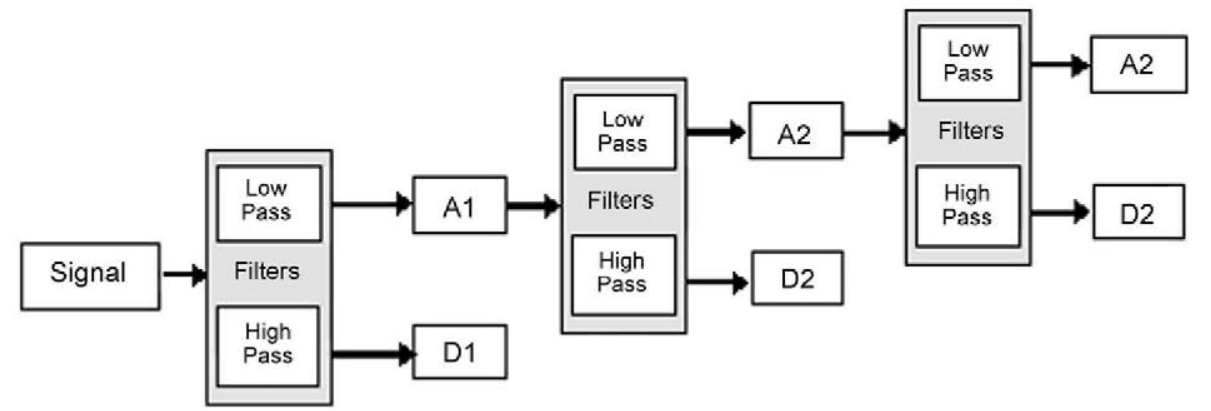

Fig. 7. Multiple-level decomposition for computing the WT.

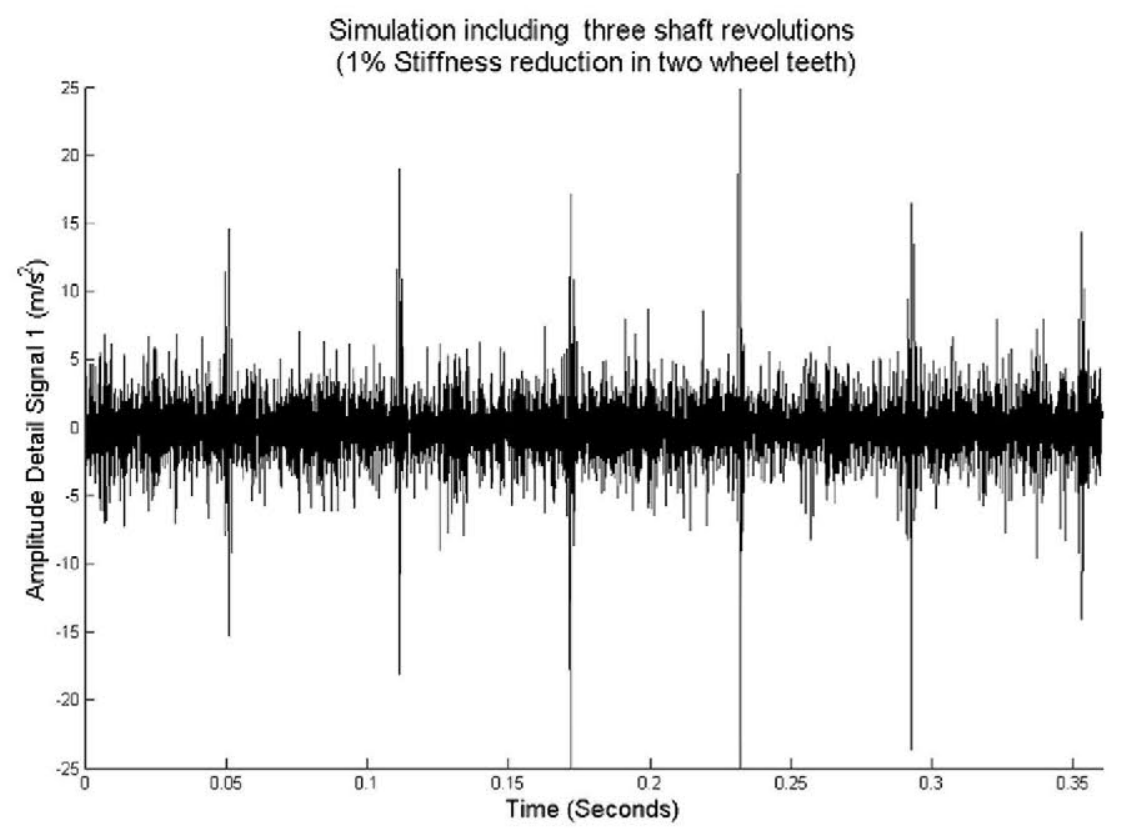

Fig. 8. Time signal corresponding to first level decompositions of a faulty vibration signals ( $1 \%$ of torsional reduction).

Notice that the stiffness reduction is introduced in a small region (short time period) of the variable mesh stiffness function in order to simulate local faults (small number of teeth affected at early stages of the damage gear).

In the studies performed in this work, ten different fault severity conditions, assumed to correspond to decreases of the averaged torsional meshing stiffness included between $1.2 \%$ and $3.2 \%$, were considered.

With the purpose of evaluating the ability of multiple detection of the proposed procedure, two different teeth were assumed to be damaged. Fig. 6 shows the acceleration responses in time domain for 'healthy' and 'damage' cases. It can be noticed that the damaged cases generate transitory changes in the response signal in a limited period of time, which are difficult to detect when only frequency techniques are used.

\section{Detection and diagnosis of faults}

The first step in the gear monitoring procedure in a rotating machine requires the fault detection. To attain this, an inspection of DWT coefficients is used as either identification or localization tool. A short description of the method is given below.

\subsection{Wavelet transforms}

The wavelet transform is the most recent solution to overcome the shortcomings of the Fourier transform. In wavelet analysis the use of a fully scalable modulated window solves the signal-cutting problem. The window is shifted along the signal and for every position the spectrum is calculated. Then this process is repeated many times with a slightly shorter (or longer) window for every new cycle. In the end the result will be a collection of time-frequency representations or multiresolution analysis of the signal, all with different resolutions. In wavelet analysis, the approximations are the high scale, low frequency components of the signal while the details are the low scale, high frequency components.

From this point of view, wavelet transforming of a signal can be regarded as passing the signal through a filter bank. In a first stage, the signal spectrum is split into two equal parts, a low-pass and a high-pass part. Both, the high-pass and the low-pass parts are half the size of the original signal and represent the high frequency (detail coefficients) and the low frequency (approximation coefficients) content of the signal, respectively. If the high-pass part contains the details we are interested in we could stop the process here. If not, the low-pass part still contains some details and therefore we can split it again. The decomposition procedure can be iterated with successive approximations being decomposed in turn until reaching the desired level of resolution (Fig. 7). In this 


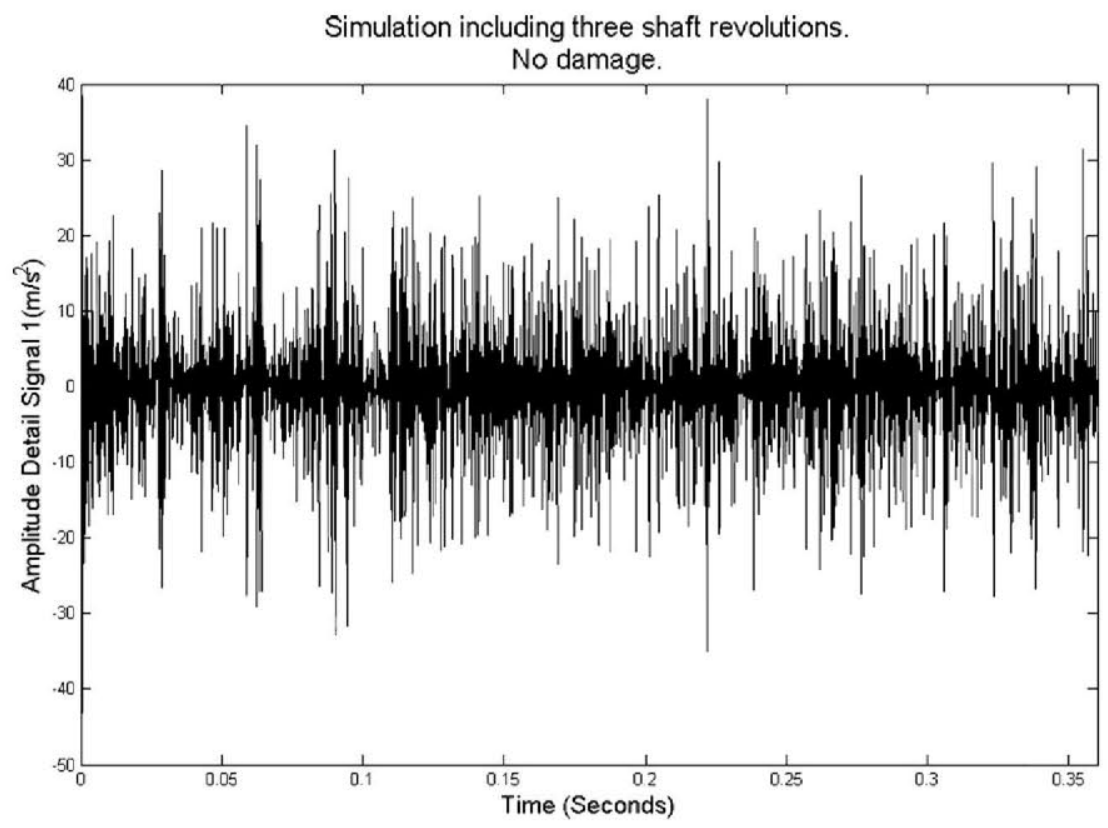

Fig. 9. Time signal corresponding to first level decompositions of a healthy signal.

way an iterated filter bank has been created. The suitable number of levels will be selected based on the nature of the signal. As different faults usually correspond to different levels of frequencies, this can be used to establish the level at which the wavelet analysis must be performed. A detailed review of WT can be found in [29].

\subsection{Signal pre-processing}

In this step it is necessary to select the most appropriate wavelet for the pre-processing as well as its level of decomposition.

To choose the appropriate family of wavelets only orthogonal and biorthogonal wavelets were considered since they permit the discrete wavelet transform to be carried out by means of the fast wavelet transform (FWT) developed by Mallat [9]. For the present study, the commonly used orthogonal set of Daubechies wavelet with eight vanishing moments ('db8') was used.
For the multiresolution analysis, three levels of decomposition were tested, but, finally, only the detail coefficients of level 1 (D1) were considered.

The fluctuation signal built by using the coefficients corresponding to the level of decomposition 1 shows clearly the presence of abnormalities and it is able to acute localize its positions per shaft revolution (Figs. 8 and 9 ).

It should be noticed that the identification of abnormalities can be performed at early stages. However, as the fault involves a short transitory period, it cannot be identified when only frequency information is used (Fig. 10).

On the other hand, it is clear from 'raw' time signals corresponding to healthy and fault vibration responses that the identification of damage is a difficult task, especially at early stages (Fig. 6).

Therefore, DWT analysis is a suitable tool for identification and localization purposes.

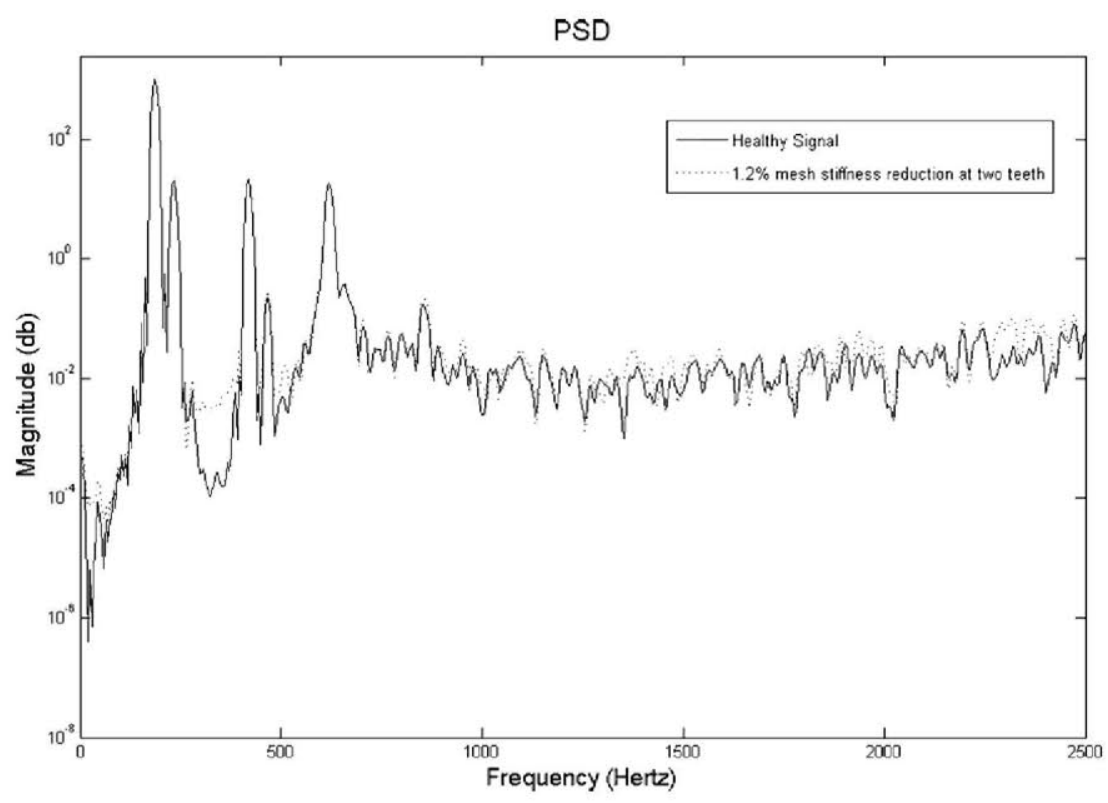

Fig. 10. PSD signals corresponding to healthy and fault vibration signal ( $1 \%$ of torsional reduction). 

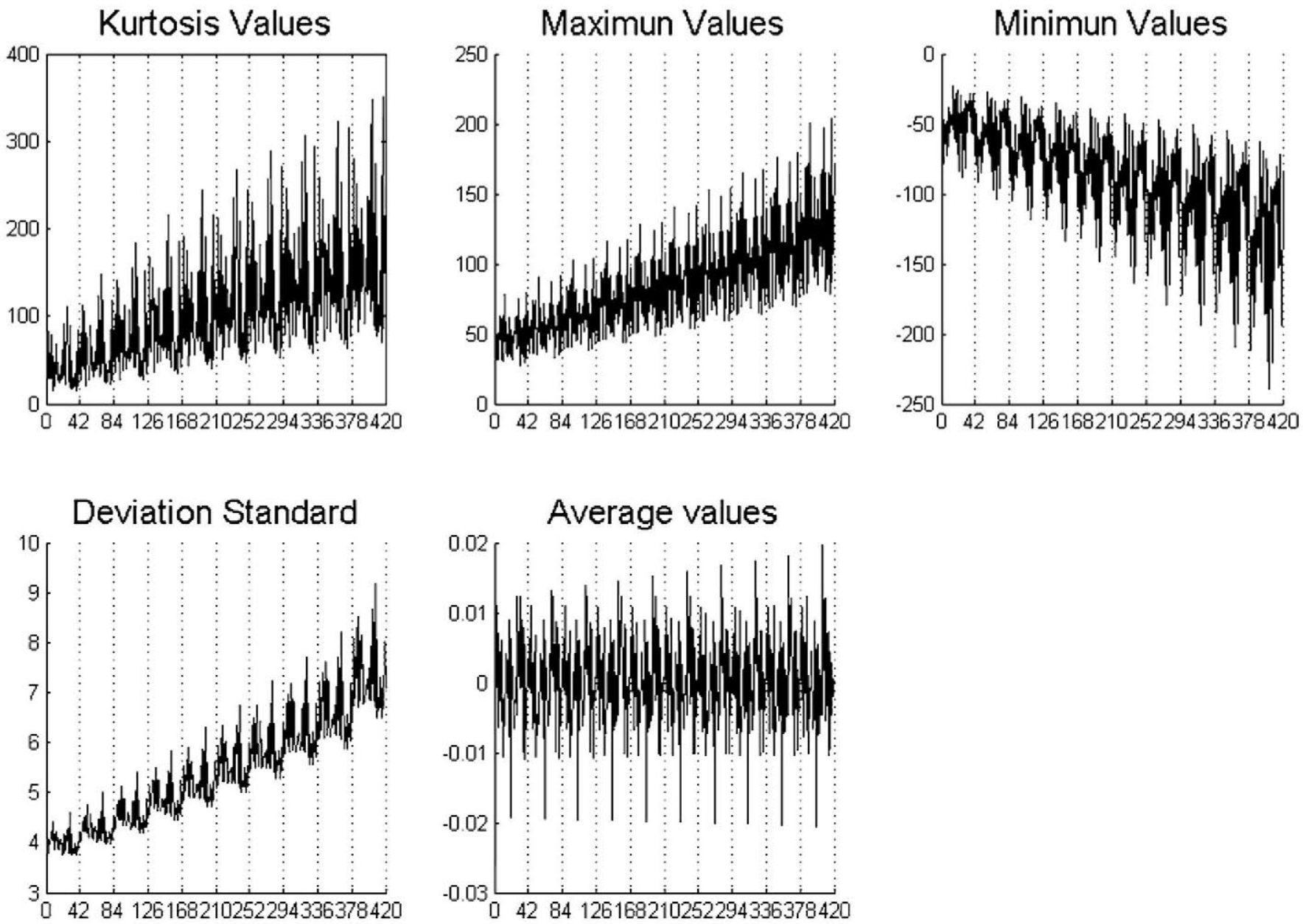

Fig. 11. Statistical features for level 1 of decomposition.

\subsection{Diagnosis robustness}

An important aspect in the development of a diagnosis tool is to evaluate its robustness to background noise.

To check this influence, a slight perturbation of the nondamaged original response signal was introduced in a couple of teeth as a consequence of a stiffness reduction; noise was not added in this case. The perturbation was introduced in an early teeth

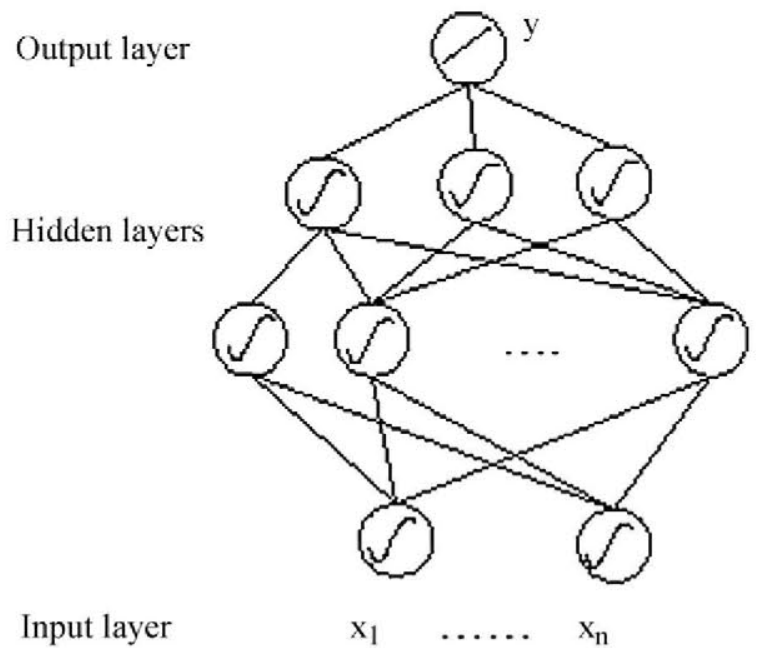

Fig. 12. MLP architecture. damaged stage (3\% of mesh stiffness reduction) generating a SNR per shaft revolution of value 1349.6. This value is clearly higher than the one used in the simulations (SNR=126.8) for a non-damaged scenario including noise. The SNR values were obtained in simulations of $15 \mathrm{~s}$.

According to these results, the perturbation induced by different damages (from $1 \%$ to $3 \%$ of stiffness reduction) would remain masked by the background noise. However, damage identification predictions were good and, therefore, it can be stated that the diagnosis tool developed in this study can be considered as a robust diagnosis procedure.

\section{Dynamic updating}

Once damage is identified in the system in a first stage, it would be helpful to update the mesh stiffness to monitor the working condition of the rotating machinery. Furthermore, this dynamic updating would make it possible to integrate the condition assessment system for predictive maintenance of the machine, which would allow extending its operative life [30,31].

Dynamic updating can be regarded as an inverse problem [32] where the target is to establish a "mapping" between direct responses in the system (in this case tangential acceleration) and mesh stiffness reduction (which can be considered as input parameter to direct problem). For this reason, to solve this inverse problem, a multi-layer perceptron neural network (MLPNN) with back-propagation training algorithm was used. In general terms, the input vector to the network was provided from the vibrational 


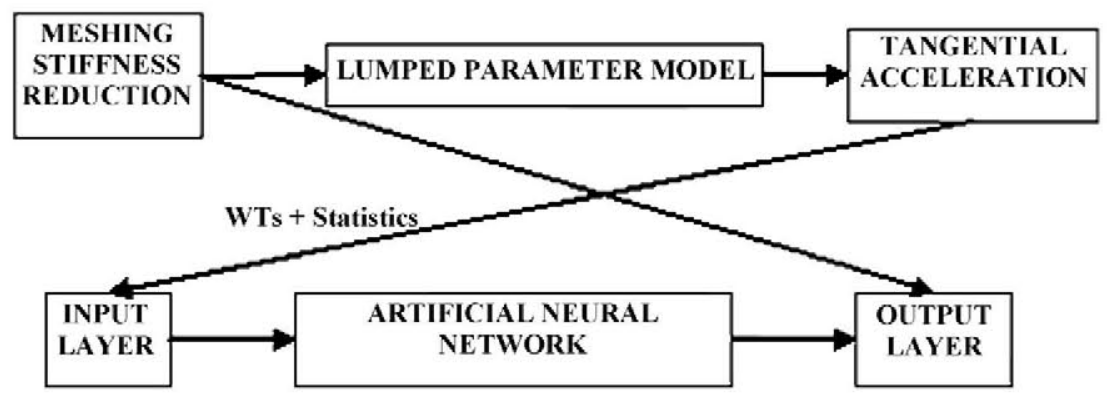

Fig. 13. MLP network training.

signal while the output layer was composed of only one node indicating the averaged torsional meshing stiffness.

The success of the procedure requires the definition as input pattern to the NN of a minimum number of features, extracted from the signal, which characterize the system conditions and are sensitive to damage. This is, in fact, the most difficult task of the process and must be studied in a very detailed way.

\subsection{Feature extraction using discrete wavelet coefficients}

In Section 4, the main features in the time-frequency domain of the tangential acceleration determined with the torsional mathematical model were extracted using DWT. In this way, the huge number of sample points obtained for every revolution of the shaft
(4252 points), necessary to ensure the stability of the integration algorithm, was clearly reduced.

However, according to the study developed in Section 4, if we take as input parameters to the MLPNN the wavelet coefficients corresponding to the level of decomposition 1 , the number of input features (2256) would still be very high. Because of this, if the "curse" of dimensionality [33] is wished to be avoided, another reduction procedure should be applied on the wavelet coefficients.

\subsubsection{Statistical characteristics of the signal}

When a perturbation is introduced in a signal transformed by the DWT, some coefficients stand out and their value depends on the number of vanishing moments of the wavelet family and also on the regularity of the perturbed signal [29]. Furthermore, the perturbation usually affects the coefficients located in the so-called
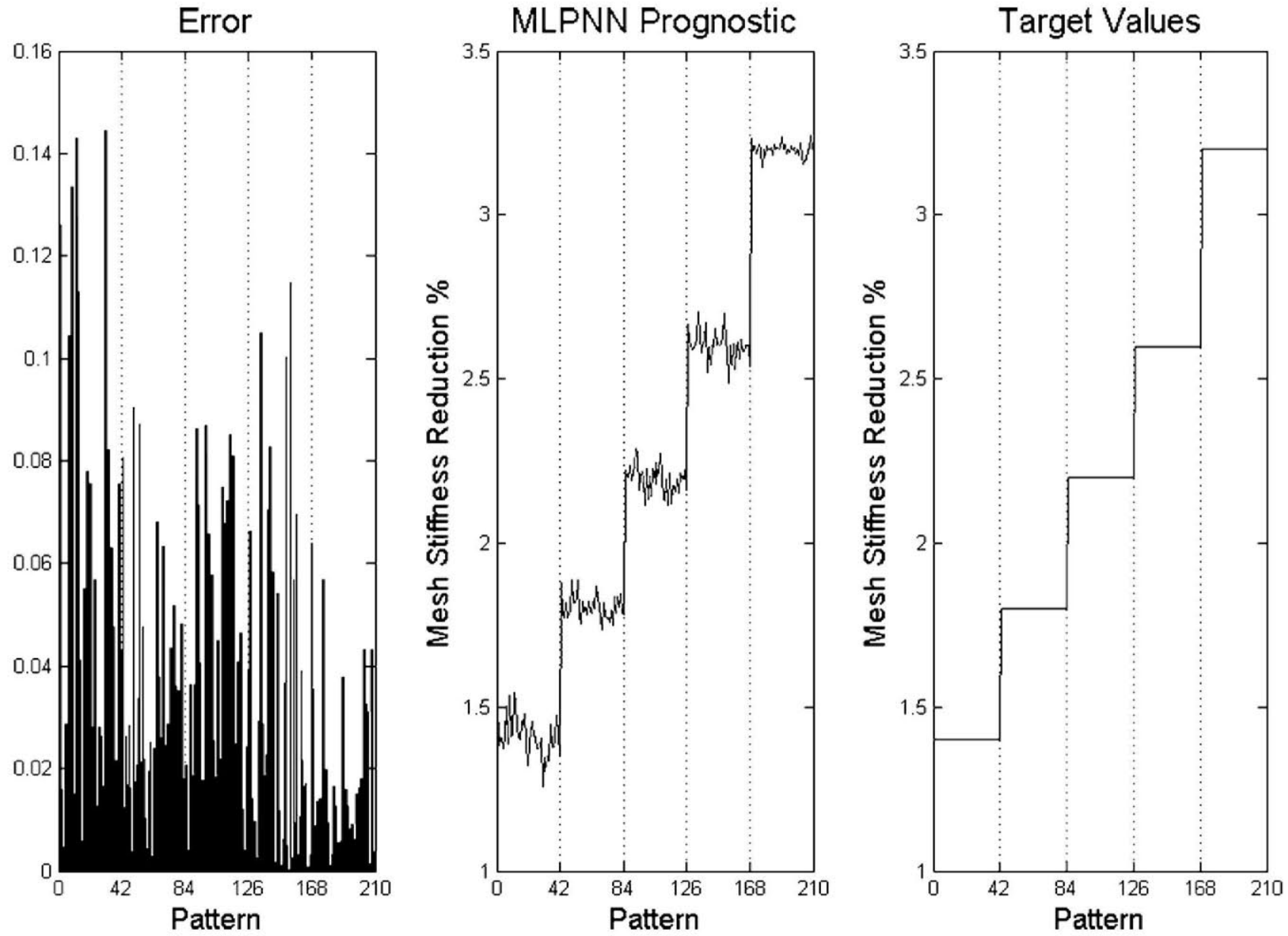

Fig. 14. Training phase simulation. 

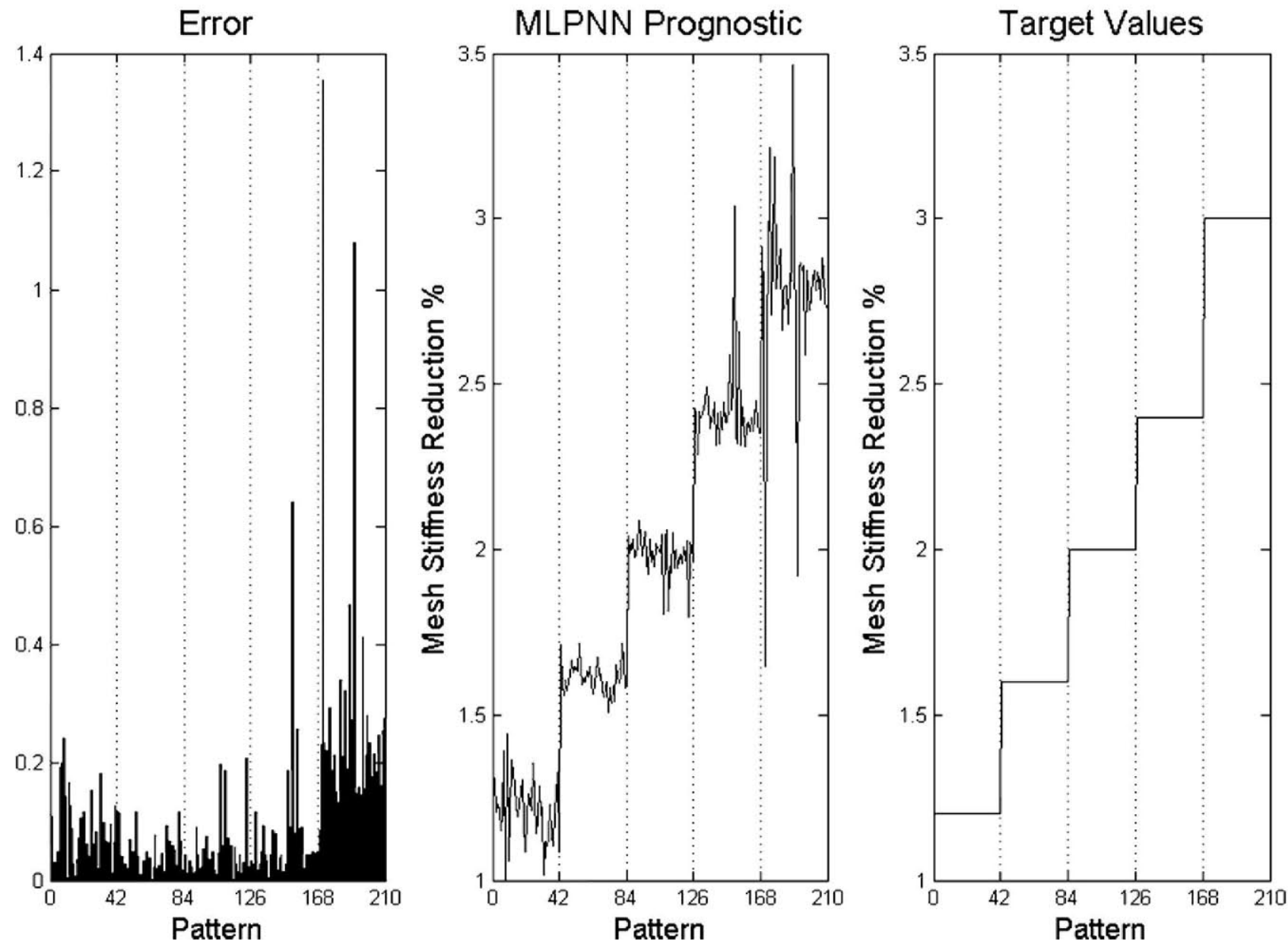

Fig. 15. Validation phase simulation.

cone of influence of the perturbation. Therefore, the peak of the coefficient reached and the width of the cone of influence are very sensitive to the regularity of the perturbation; these properties can be used to quantify damage.

The standard deviation from the mean is used widely in statistics to indicate the degree of dispersion while kurtosis, which is the normalized fourth moment, gives an indication about the spikiness of the analyzed data. For this reason, it is expected that when a perturbation is introduced on a signal, either the kurtosis or the standard deviation will be seriously affected. The higher the peak of the perturbation the higher the kurtosis value and, furthermore, as the width of the cone of influence grows with the damage severity, the standard deviation will do so as well.

Fig. 11 shows the maximum, minimum, mean, standard deviation and kurtosis values obtained from the wavelet coefficients associated to each pattern for the level of decomposition 1 . In these figures, 420 patterns were represented every 42 of them corresponding to the same reduction of the torsional meshing stiffness beginning with $1.2 \%$ and ending with $3.2 \%$ with increments of $0.2 \%$. It should be remembered that each pattern corresponds to one wheel revolution and, therefore, the statistical parameters are extracted using DWT coefficients obtained in every revolution and for every level of decomposition.

As can be observed from Fig. 11 these statistical parameters, except the mean value, showed sensitive to the decrease in mesh stiffness and, therefore, might be selected as input to the MLPNN. Therefore, if, as in our case, only the level 1 of decomposition were considered after the statistical study, the NN would be configured by only four input neurons, which represents a very noticeable reduction.

\subsection{Dynamic updating using multilayer perceptron neural networks}

\subsubsection{Network configuration and training}

A multilayer perceptron neural network was used in the present work to identify the damage severity. MLPNNs consist of an input layer of source nodes, one or more hidden layers of computation nodes and an output layer (Fig. 12). As has been commented previously, maximum, minimum, standard deviation and kurtosis values obtained from the wavelet coefficients for the level of decomposition selected by being sensitive enough to damage are adopted as the input vector to the perceptron neural network. On the other hand, the output of the network is only one value representing the change in the averaged torsional meshing stiffness.

The number of hidden layers and the number of nodes in each hidden layer affect the generalization capability of the network. It has been demonstrated that the number of hidden layers in a regression problem has to be higher than two [34,35]; therefore this is the minimum number that should be used as a starting point. With that restriction, a heuristic method has been applied to select the number of hidden layers and the nodes.

The training of the MLP network (Fig. 13) involves finding values of the connection weights, which minimize an error function between the actual network output and the corresponding target value in the training set. The training algorithm used is based on 
resilient propagation [36]. The weight adjust is performed by minimizing the mean square error function. This error function usually corresponds to a way of quantifying the deviation with respect to the target values.

The data needed for network training are generated from the lumped parameter model of Section 3. After completing the network training, new patterns generated with the lumped model are used to check the validity of the network.

\subsubsection{Quantification results}

The designed network has two hidden layers with 20 and 5 neurons, respectively. In the training phase, a total number of 210 shaft revolutions (patterns) were obtained by introducing in the lumped gear model five different decrease levels $(1.4 \%, 1.8 \%, 2.2 \%, 2.6 \%$ and $3.2 \%$ ) of the meshing stiffness. Furthermore, to be more consistent with experimental reality, white noise was added to the drive torque with the same characteristics as in Section 3. This allows different training patterns for the same damage severity.

Net prognostics of the meshing modulation stiffness together with the target values during the training phase are shown in Fig. 14. In the same way, errors between predictions and real values during this phase are depicted for every level of severity in the first column of the same figure. To define the error, a function has been established as follows:

$E R R O R=100\left[\frac{a b S\left(K_{R E A L}-K_{\text {ESTIMATED }}\right)}{K_{\text {REAL }}}\right]$

In the above expression the prefix $K$ indicates the mesh torsional stiffness, either estimated by the neural network or the real value, and $a b s$ the absolute operator.

The training has been performed every 42 damage patterns for each level of mesh stiffness reduction $(1.4 \%, 1.8 \%, 2.2 \%, 2.6 \%$ and $3.2 \%$ ), and every prognostic corresponds to one shaft revolution $(0.24 \mathrm{~s})$. The averaged error value for all the training patterns is below $0.03 \%$ which represents a very small quantity when compared to the meshing stiffness variations taken into account.

After completing the network training, 210 new patterns generated with the lumped model with damage extents $(1.2 \%, 1.6 \%$, $2.0 \%, 2.4 \%$ and $3.0 \%$ ) different from the training ones were fed into the trained network in order to evaluate how well the proposed approach works, i.e. how significant the generalization ability is for predicting damage severities. In the same way as for the training stage, the comparison between predictions and real values of the meshing stiffness for each level of severity are shown in Fig. 15 and the errors between predicted and target values are shown in the first column of Fig. 15.

In this case the total averaged error value is below $0.09 \%$, which is slightly higher than the value observed in the training stage; however, these values are close enough to affirm that the proposed approach generalizes well and can be used as a tool to track the evolution of the reduction of the meshing stiffness which is a straight indicator of the damage progress.

\section{Conclusions}

In this paper, a two-stage procedure for the gear dynamic monitoring in rotating machinery from vibration analysis has been developed. Both stages are based on the combined use of wavelet transforms and neural networks. The first stage, fault detection and diagnosis, combines the capabilities of wavelet transform. To this first stage, a new stage was added for estimating the mesh stiffness reduction. To achieve this, a multilayer perceptron neural network was used. This network was fed with statistical parameters obtained from the wavelet coefficients derived only for the most sensitive levels of decomposition to damage. The output of the network is only one value representing the drop in the averaged torsional meshing stiffness when a failure appears. This value is highly related with local failure and is a clear indicator of damage evolution. It should be remarked that this algorithm could be implemented in another gearbox machine following the steps given in Section 2. Therefore it is a generalized diagnostic and dynamic updating tool for monitor the gear dynamics.

The algorithm proposed in this paper allows performing a diagnostic at early stages of the mesh stiffness variation when a local damage appears (it is able to detect a $1 \%$ mesh stiffness decrease). Detection, localization and dynamic updating are predicted and provided very soon and every shaft revolution, nearly on-line, therefore this system can be used as a powerful tool in order to avoid catastrophic failures in gears caused when critical operating points (speed and torque) are exciting some potential risk resonances modified by new mesh stiffness as a consequence of local gear damage.

This mesh stiffness updating is a key aspect of the monitoring gear dynamics because it allows knowing nearly on-line (per shaft revolution) critical parameters such as load carrying capacity of the gear and dynamic tooth loads.

The errors obtained during the dynamic updating stage can be considered negligible when compared to the changes in the torsional stiffness causing the faults; therefore, the procedure can be considered as suitable for dynamic monitoring purposes.

The proposed procedure allows for a suitable scheduling of timely corrective and repair actions on rotating machinery. Furthermore, it allows establishing the best working condition, referred to torque and speed, for a damaged rotary machine, when a failure takes place. In conjunction with this procedure it would be very interesting to relate the type of specific local failure (size of spalls in tooth flank, crack length, etc.) with local mesh stiffness variations in order to build a complete prognosis tool. However, this would require a thoroughly study of the specific failure mechanism and the aim of the study developed here was to implement a procedure for dynamic condition monitoring in machines with gear local failures characterized by a mesh stiffness reduction.

\section{Acknowledgement}

The writers acknowledge support for the work reported in this paper from the Ministry of Science and Innovation of Spain (Project BIA2010-20234-C03-01).

\section{References}

[1] A. Parey, M. Badaoui, F. Guillet, N. Tandon, Dynamic modelling of spur gear pair and application of empirical mode decomposition-based statistical analysis for early detection of localized tooth defect, Journal of Sound and Vibration 294 (2006) 547-561.

[2] G. Dalpiaz, A. Rivola, R. Rubini, Effectiveness and sensitivity of vibration processing techniques for local fault detection in gears, Mechanical Systems and Signal Processing $14(2000) 387-412$.

[3] W.Q. Wang, F. Ismail, M.F. Golnaraghi, Assessment of gear damage monitoring techniques using vibration measurements, Mechanical Systems and Signal Processing 15 (2001) 905-922.

[4] D. Gabor, Theory of communication, IEEE Journal 93 (1946) 429-457.

[5] C.W. Hoelst röm, An expansion of a signal in Gaussian elementary signals, IEEE Transactions on Information Theory 12 (1966) 81-82.

[6] W.J. Staszewski, K. Worden, G.R. Tomlinson, Time-frequency analysis in gearbox fault detection using the Wigner-Ville distribution and pattern recognition, Mechanical Systems and Signal Processing 11 (1997) 673-692.

[7] H.I. Choi, W.J. Williams, Improved time-frequency representation of multicomponent signals using exponential kernels, IEEE Transactions on Acoustics Speech and Signal Processing 37 (1989) 862-871.

[8] I. Daubechies, Orthonormal bases of compactly supported wavelets, Communications on Pure and Applied Mathematics XLI (1988) 909-996.

[9] S.G. Mallat, Theory for multiresolution signal decomposition: the wavelet represent ation, IEEE Transactions on Pattern Analysis and Machine Intelligence 11 (1989) 674-693.

[10] W.J. Wang, P.D. McFadden, Application of wavelets togearbox vibration signals for fault detection, Journal of Sound and Vibration 192 (1996) 927-939. 
[11] D. Boulahbal, M.F. Golnaghari, F. Ismail, Amplitude and phase wavelet maps for the detection of cracks in geared systems, Mechanical Systems and Signal Processing 13 (1999) 423-436.

[12] J. Sanz, R. Perera, C. Huerta, Fault diagnosis of rotating machinery based on auto-associative neural networks and wavelet transforms, Journal of Sound and Vibration 302 (2007) 981-999.

[13] A.V. Ovanesova, L.E. Suarez, Applications of wavelet transforms to damage detection in frame structures, Engineering Structure 26 (2004) 39-49.

[14] J. Han, W.X. Ren, Z. Sun, Wavelet packet based damage identification of beam structures, International Journal of Solids and Structures 42 (2005) 6610-6627.

[15] K. Gurley, A. Kareem, Application of wavelet transforms in earthquake, wind and ocean engineering, Engineering Structure 21 (1999) 149-167.

[16] H.N. Ozguben, D.R. Houser, Mathematical models used in gear dynamics-a review, Journal of Sound and Vibration 121 (1988) 383-411.

[17] I. Yesilyurt, F. Gu, A.D. Ball, Gear tooth stiffness reduction measurement using modal analysis and its use in wear fault severity assessment of spur gears, NDTE\&E International 36 (2003) 357-372.

[18] A. Saxena, A. Saad, Evolving an artificial neural network classifier for condition monitoring of rotating mechanical systems, Applied Soft Computing 7 (2007) $441-454$.

[19] E. Zio, P. Baraldi, G. Gola, Feature-based classifier ensembles for diagnos ing multiple faults in rotating machinery, Applied Soft Computing 8 (2008) $1365-1380$.

[20] J. Yu, A hybrid feature selection scheme and self-organizing map model for machine health assessment, Applied Soft Computing 11 (2011) 4041-4054.

[21] P. Konar, P. Chattopadhyay, Bearing fault detection of induction motor using wavelet and Support Vector Machines (SVMs), Applied Soft Computing 11 (2011) 4203-4211.

[22] A. Parey, N. Tandon, Spur gear dynamic models including defects - a review, The Shock and Vibration Digest 35 (2003) 465-478.

[23] P.D. McFadden, Detecting fatigue cracks in gears by amplitude and phase demodulation of the meshing vibration, Journal of Vibration Acoustics Stress and Reliability in Design - Transactions of the ASME 108 (1986) 165-170.

[24] F. Kong, A combined method for triplex pump fault diagnosis based on wavelet transform, fuzzy logic and neuro-networks, Mechanical Systems and Signal Processing 18 (2004) 161-168.

[25] J.Y.Z. He, Y. Zi, Gear fault detection using customized multiwavelet lifting schemes, Mechanical Systems and Signal Processing 24 (2010) 1509-1528.

[26] G. Dalpiaz, A. Rivola, R. Rubini, A kineto-elastodynamic model of a gear testing machine, in: Proc. Int. Conf. on Mechanical Transmissions and Mechanisms, MTM'97, Tianjin, China, 1997, pp. 549-553.

[27] G. Dalpiaz, Early detection of fatigue cracks in gears by vibrations analysis techniques, Österreichische Ingenieur und Architekten Zeitschrift (ÖIAZ) 135 (1990) 312-317.

[28] P.J. Prince, J.R. Dormand, High order embedded Runge-Kutta formulae, Journal of Computational and Applied Mathematics 7 (1981) 67-75.
[29] S.G. Mallat, A Wavelet Tour of Signal Processing, Academic Press, 1999.

[30] M.J. Roemer, G.J. Kacpryznski, R.F. Orsagh, B.R. Marshall, Prognosis of rotating machinery components, in: D.J. Inman, C.R. Farrar, V. Lopes, V. Steffen (Eds.) Damage Prognosis for Aerospace, Civil and Mechanical Systems, Wiley, 1995, pp. $385-420$.

[31] C.R. Farrar, N.A.J. Lieven, Damage prognosis: the future of structural health monitoring, Philosophical Transactions of the Royal Society A 365 (2007) 623-632.

[32] A. Tarantola, Inverse Problem Theory and Methods for Parameter Estimation, SIAM, 2005.

[33] C.M. Bishop, Neural Networks for Pattern Recognition, Oxford University Press, 1995.

[34] L.K. Jones, Constructive approximations for neural networks by sigmoidal functions, Proceedings of the IEEE 78 (1990) 1586-1589.

[35] E.K. Blum, L.K. Li, Approximation theory and feedforward networks, Neural Networks 4 (1991) 511-515.

[36] M. Riedmiller, H. Braun, A direct method for faster backpropagation learning: the RPROP algorithm, in: Proc. IEEE Int. Conf. on Neural Networks, 1993, pp. 586-591.

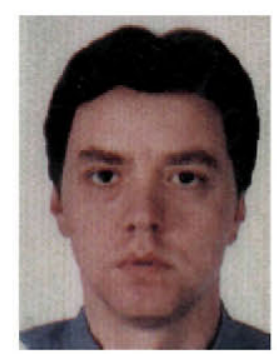

Ricardo Perera is Full Professor and Head of the Department of Structural Mechanics for the Technical University of Madrid. He got the Ph Degree on 1995 and from then he has coordinated many research projects related to topic such as material modeling, strengthening of concrete structures with FRP composites, structural health monitoring in mechanical and civil engineering and soft computing. He has published more than 36 journal papers about these topics and has participated actively in numerous conferences and seminars.

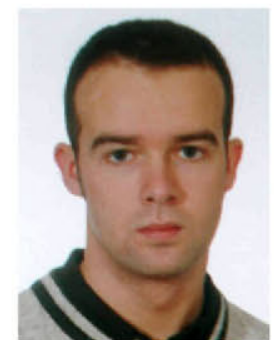

Javier Sanz is a Mechanical Engineer who got the Ph Degree on 2007. His main research focuses on structura dynamics, damage diagnosis in rotating mechanical sys tems, wavelets and soft computing. At present he is in charge of drive train modeling and integration in general multi-body of wind turbine in Acciona Windpower. 